\title{
A Study on Microalbuminuria in Non-Diabetic Hypertensive Patients Attending a Tertiary Care Hospital in South India
}

\author{
Sunil Abdul Salim ${ }^{1}$, Sajan Christopher ${ }^{2}$ \\ 1,2 Department of General Medicine, Government Medical College, Thiruvananthapuram, Kerala, India.
}

\section{ABSTRACT}

\section{BACKGROUND}

Essential hypertension represents 90 - $95 \%$ of all instances of hypertension. Microalbuminuria was observed to be related to increased chances of coronary artery disease even in patients already on treatment for hypertension. Microalbuminuria was identified with left ventricular hypertrophy recommending equal heart harm and albuminuria in hypertensive patients. However, the pervasiveness of hypertension is high in India, the connection between microalbuminuria and target organ damage in hypertension isn't well studied. This study intended to decide the extent of microalbuminuria in non-diabetic patients with essential hypertension and concentrate on the relationship of microalbuminuria with the worsening of left ventricular hypertrophy (LVH) and hypertensive retinopathy (HRP).

\section{METHODS}

This was a hospital-based cross-sectional analysis conducted in a hospital from January 2019 to September 2019. Information was gathered from 93 patients fulfilling the consideration standards utilizing a formerly planned semi-structured questionnaire. Urine microalbumin level, echocardiography, ECG and fundus assessment were done. The study variables were recorded and dissected utilizing SPSS software.

\section{RESULTS}

The mean age of the group populace was $55 \pm 8.89$ years. Microalbuminuria was present in $68 \%$. There was a measurably critical relationship between the age group studied and the predominance of microalbuminuria. There was a huge factual affiliation between the presence of microalbuminuria and LVH in hypertensive patients $(\mathrm{P}<0.0001)$, with microalbuminuria and HRP $(\mathrm{P}<0.0001)$ and with microalbuminuria and long-standing hypertensives $(\mathrm{P}<0.0001)$.

\section{CONCLUSIONS}

The larger part of microalbuminuria positives was found disseminated among higher ages. There was a positive relationship among patients with microalbuminuria and $\mathrm{LVH}$, with retinopathy in hypertensive patients, with grade 2 hypertension and with long-standing hypertensives.

\section{KEY WORDS}

Hypertension, Microalbuminuria, LVH, Hypertensive Retinopathy.
Corresponding Author: Dr. Sajan Christopher, H. No. 51 (1), Christina, Watts Lane, Nanthencode, Thiruvananthapuram-695003, Kerala, India.

E-mail: sajanchristopher74@gmail.com

DOI: $10.14260 /$ jemds/2021/743

How to Cite This Article:

Salim SA, Christopher S. A study on microalbuminuria in non-diabetic hypertensive patients attending a tertiary care hospital in South India. J Evolution Med Dent Sci 2021;10(43):3665-3669, DOI: $10.14260 /$ jemds/2021/743

Submission 06-09-2021, Peer Review 14-10-2021, Acceptance 21-10-2021, Published 25-10-2021.

Copyright (c) 2021 Sunil Abdul Salim et al. This is an open access article distributed under Creative Commons Attribution License [Attribution 4.0 International (CC BY 4.0)] 


\section{BACKGROUND}

Primary hypertension accounts for almost all cases of hypertension. ${ }^{1}$ Although, hypertension is often without any symptoms, the disease is related to multiple target organ damage manifestations and associated clinical conditions. ${ }^{2}$ Cardiovascular risk rises steeply with different grades of hypertension. This risk increases further when additional risk factors accumulate. Microalbuminuria, hypertensive retinopathy and left ventricular hypertrophy (LVH) are important predictors of cardiovascular morbidity and mortality. $^{2}$ A significant proportion of the hypertensive population shows microalbuminuria in which there occurs a slight elevation in the urinary albumin excretion above a preset threshold. Microalbuminuria is one of the earliest markers of renal injury and vascular dysfunction. These people are more prone to develop cardiovascular and renal complications in later life. ${ }^{3}$ Microalbuminuria is defined as a persistent elevation of albumin in the urine from greater than 30 to less than $300 \mathrm{mg} /$ day. This may not be detectable with routine protein dipstick assays. ${ }^{1}$ Microalbuminuria is an index of increased renal endothelial permeability and diffuse endothelial dysfunction.4,5 Several studies show that a positive correlation exists between microalbuminuria and elevated blood pressure in hypertensive patients. As most hypertensive patients are almost always asymptomatic, they may be unaware of the consequent progressive damage to various target organs for as long as 10 to 20 years. If microalbuminuria is positively associated with hypertension, its presence calls for more strict control of blood pressure to prevent future cardiovascular risks. Evidence from the literature suggests that even very low levels of microalbuminuria with albumin excretion rates as low as 4.8 $\mu \mathrm{g} / \mathrm{min}$ is associated with increased risk of cardiovascular and retinovascular disease, independent of the presence of other risk factors. ${ }^{4}$ So we intended to find the proportion of microalbuminuria in non-diabetic patients with essential hypertension and find if there was any association of it with the development of left ventricular hypertrophy and hypertensive retinopathy.

\section{METHODS}

This study was approved by the Institutional review board and Institutional ethics committee and was conducted from January 2019 to September 2019 at a tertiary care medical college catering for nearly 10000 inpatients daily. It was a hospital-based cross-sectional study done among nondiabetic essential hypertensive in-patients with blood pressure above $140 / 90 \mathrm{~mm}$ of $\mathrm{Hg}$ and aged more than 30 years of either gender and admitted in general medicine ward of a tertiary care teaching institution in South India. Unwilling patients, diabetic patients, patients with renal disease, heart failure, macroalbuminuria and those with a positive history or clinical signs of ischaemic heart disease were excluded from the study. The sample size was calculated according to the formula $4 \mathrm{PQ} / \mathrm{L} 2$ where; $\mathrm{P}$ is the prevalence, $\mathrm{Q}$ is 100 Prevalence, and $\mathrm{L}$ is an allowable error. From a study conducted by Sharan Badiger, ${ }^{6}$ where the prevalence of microalbuminuria in hypertensive patients was $63 \%$, the sample size was calculated to be 93. All consecutive patients who satisfied the selection criteria were selected. The study tool used was a previously designed semi-structured questionnaire. Study variables included microalbuminuria, left ventricular hypertrophy (LVH), hypertensive retinopathy (HRT), duration of hypertension and sociodemographic variables like age and gender. Informed written consent was taken from the study patients before they were enrolled for the study. Relevant details of the patients were collected using a semi-structured questionnaire. Urine microalbumin was tested in a urine spot sample. Echocardiography, ECG interpretation and fundus examination were done in the Department of Internal Medicine.

Hypertension is defined as office systolic BP (SBP) values $>140 \mathrm{mmHg}$ and/or diastolic BP (DBP) values > $90 \mathrm{mmHg}$. The 2018 ESC/ESH Guidelines for the management of arterial hypertension ${ }^{7}$ state that there are 3 grades of hypertension -

- $\quad$ Grade 1: SBP 140-159 mm of Hg and/or DBP 90-99 mm of $\mathrm{Hg}$.

- Grade 2: SBP 160-179 mm of Hg and/or DBP 100-109 $\mathrm{mm}$ of $\mathrm{Hg}$.

- Grade 3: SBP > $180 \mathrm{~mm}$ of $\mathrm{Hg}$ and/or DBP > $110 \mathrm{~mm}$ of $\mathrm{Hg}$.

A person is said to have microalbuminuria if his albumin to creatinine ratio is in the range of $30-300 \mathrm{mg} / \mathrm{g}$ creatinine in a urine spot sample. ${ }^{8}$

Left ventricular hypertrophy was diagnosed by echocardiography when left ventricular posterior wall or interventricular septal thickness was greater than $1.3 \mathrm{~mm}^{9}$

All the patients were subjected to direct fundoscopy to assess retinal vasculature. It was also used to examine the optic disc for signs of hypertensive retinopathy. Keith Wagener Barker classification ${ }^{10}$ was used for grading of retinopathy. Grade 1 and 2 were considered as early retinopathic changes whereas grades 3 and 4 were considered as advanced retinopathy.

\section{Statistical Analysis}

Data collected were entered in SPSS software. Quantitative variables were expressed as means. Qualitative variables were expressed as proportions. Association of outcome variables like left ventricular hypertrophy and hypertensive retinopathy were analysed using the chi-square test.

\section{RESULTS}

During the eight months, 93 patients who were admitted for various reasons and had a blood pressure reading above $140 / 90 \mathrm{~mm}$ of Hg were enrolled for the study after careful analysis of the exclusion criteria. Most of them were above 50 years and the mean age of the group studied was $55 \pm 8.89$ years. Of this, the mean age in the males was $56.6 \pm 8.9$ years and that of the females was $52.6 \pm 7.0$ years. The majority of the cases with microalbuminuria was found in the higher age group, $59.75 \pm 16.32$ years in males versus $57.9 \pm 17.58$ years in females. There was male sex preponderance in our study with $65 \%$ (60 patients) of them being males and $35 \%$ (33 patients) females. 
In the study population, 63 patients (68\%) had associated microalbuminuria. Of this, 44 patients (47\%) were males and 19 patients (21\%) were females. Although the prevalence of microalbuminuria was more in males when compared to females (47 \% Vs $21 \%$ ), no significant association was present between gender and microalbuminuria (P-value 0.0924) (Table 1).

\begin{tabular}{|cccc|}
\hline Gender & MAU Positive & MAU Negative & Total \\
Male & 44 & 16 & $60(65 \%)$ \\
Female & 19 & 14 & $33(35 \%)$ \\
Total & $\mathbf{6 3 ( 6 8 \% )}$ & $\mathbf{3 0}(\mathbf{3 2} \%)$ & $\mathbf{9 3 ( 1 0 0 \% )}$ \\
\hline \multicolumn{4}{|c|}{ Table $\mathbf{1 .}$ Baseline Gender Characteristics } \\
\hline \multicolumn{4}{|c|}{ Footnote: MAU: Microalbuminuria (P value 0.0924) } \\
\hline \multicolumn{4}{|c}{}
\end{tabular}

LVH was present in 49 people. Of this, 43 people (88\%) had associated microalbuminuria. The association of LVH with microalbuminuria was studied. A significant statistical association was noted between the presence of microalbuminuria and LVH $(\mathrm{P}<.0001)$ which is discussed in table 2 .

\begin{tabular}{|ccc|}
\hline MAU & LVH Present & LVH Absent \\
\hline MAU Positive & 43 & 20 \\
MAU Negative & 6 & 24 \\
Total & $\mathbf{4 9}(\mathbf{5 2 . 7} \%)$ & $\mathbf{4 4}(\mathbf{4 7 . 3 1} \%)$ \\
\hline Table & 2. Left Ventricular Hypertrophy $(\mathbf{L V H})$ & and Microalbuminuria \\
\hline Footnote: MAU: Microalbuminuria P<0.0001 & \\
\hline
\end{tabular}

48 patients (51.7\%) had evidence of retinopathy. Of this, $87.5 \%$ had microalbuminuria. Microalbuminuria was higher in patients with early retinopathy $(66.7 \%)$ than in those with advanced retinopathy (20.8\%) (32 patients Vs 10 patients). A significant statistical association was also noted between people with microalbuminuria and those with hypertensive retinopathy $(\mathrm{P}<0.001)$. This has been documented in Table 3.

\begin{tabular}{|cccc|}
\hline MAU & $\begin{array}{c}\text { Early Retinopathy } \\
\text { (Grade 1 and 2) }\end{array}$ & $\begin{array}{c}\text { Advanced Retinopathy } \\
\text { (Grade 3 and 4) }\end{array}$ & $\begin{array}{c}\text { Total N } \\
\text { (\%) }\end{array}$ \\
MAU positive & $32(66.7 \%)$ & $10(20.8 \%)$ & $42(87.5 \%)$ \\
MAU negative & $5(10.4 \%)$ & $1(2.1 \%)$ & $6(12.5 \%)$ \\
Total & $\mathbf{3 7 ( 7 7 . 1 \% )}$ & $\mathbf{1 1}(\mathbf{2 2 . 9 \% )}$ & $\mathbf{4 8}$ \\
\hline Table 3. Hypertensive Retinopathy and Microalbuminuria \\
\hline Footnote: MAU: Microalbuminuria.(P<0.0001) \\
\hline
\end{tabular}

Microalbuminuria and its association with the duration and severity of hypertension were studied. For comparing the duration of hypertension, the study population was divided into two groups - Long-standing hypertensives (i.e. > 15 years) and those of short duration (i.e. $<15$ years). We found that 12 patients $(12.9 \%)$ with a shorter duration of hypertension had microalbuminuria whereas 51 (54.8\%) long-standing hypertensives had microalbuminuria. As the duration of hypertension increases, the prevalence of microalbuminuria also increases (54.8 \% Vs 12.9 \%). A significant statistical and clinical association was also established between the duration of hypertension and the presence of microalbuminuria. $\mathrm{P}<0.0001$.

\begin{tabular}{|ccc|}
\hline $\begin{array}{c}\text { Duration Since the } \\
\text { Detection of } \\
\text { Hypertension }\end{array}$ & Microalbuminiuria & $\begin{array}{c}\text { No } \\
\text { Microalbuminuria }\end{array}$ \\
$<15$ years & $12(12.9 \%)$ & $22(23.7 \%)$ \\
$>15$ years & $51(54.8 \%)$ & $8(8.6 \%)$ \\
Total & $\mathbf{6 3}$ & $\mathbf{3 0}$ \\
\hline Table 4. Microalbuminuria and Duration of Hypertension \\
\hline Footnote: MAU: Microalbuminuria $(P<0.0001)$. \\
\hline
\end{tabular}

Hypertension severity and its association with microalbuminuria were studied. The study population was divided based on the severity of hypertension into three groups i.e., Grade 1-3. Of the 58 people with grade 2 hypertension, 42 patients $(45.2 \%)$ had associated microalbuminuria. Most of the people in the study group (i.e., 58 patients) had grade 2 hypertension and there was a positive association between microalbuminuria and grade 2 hypertensives $(P=0.002)$. (Table 5$)$. This association was not noted in grade 1 and grade 3 hypertension. $(\mathrm{P}=0.942,0.118)$

\begin{tabular}{|c|c|c|c|}
\hline $\begin{array}{l}\text { Hypertension } \\
\text { Severity }\end{array}$ & $\begin{array}{c}\text { Microalbuminuria } \\
(\%)\end{array}$ & $\begin{array}{c}\text { No Microalbuminuria } \\
(\%)\end{array}$ & $\begin{array}{c}P \\
\text { Value }\end{array}$ \\
\hline Grade 1 & 15 (16.1\%) & 13 (13.9\%) & 0.942 \\
\hline Grade 2 & $42(45.2 \%)$ & $16(17.2 \%)$ & 0.001 \\
\hline Grade 3 & $6(6.5 \%)$ & $1(1.1 \%)$ & 0.118 \\
\hline
\end{tabular}

\section{DISCUSSION}

Our study correlated well with other studies on many levels. Most of the people were middle-aged and the mean age was $55 \pm 8.89$ years. Our study continues to show that there is still an increased prevalence of essential hypertension in middleaged people although there is upcoming evidence of young hypertensives in other parts of India. Gender-wise, our study suggests that females are developing hypertension at early ages compared to men (52.6 years Vs 56.6 years) probably due to the early onset of menopause in females. There was slightly increased age preponderance in males. In spite of this, gender did not pose any higher risk for the presence of microalbuminuria.

In our study, microalbuminuria was seen in $68 \%$ of the patients. This was similar to studies conducted by Poudyal et al. (62.5 \%), ${ }^{11}$ Rameez et al. ${ }^{12}$ (69 \%) but much higher than the study by Stalin et al. ${ }^{13}(24.3 \%)$ and Hitha et al. $(26.67$ \%). ${ }^{14}$ Studies from Nigeria as in Busari et al. ${ }^{15}$ showed a lower prevalence of microalbuminuria (32.2\%) and a study in the Iranian population by Hemmati et al. ${ }^{16}$ showed a much lower prevalence of $5.6 \%$. Variations in different studies conducted could be due to different criteria used in selection, inpatients versus outpatients in the study population, differences in the methods used for detecting microalbuminuria, the severity of hypertension, different age, population and ethnicity, and coexistent comorbidities.

People with increasing age are more likely to have microalbuminuria in our study also, as observed in other studies like Wachtell et al..$^{3}$ (beta $=.095, \mathrm{P}<.01$ ) and Stalin et al. ${ }^{13}$ As expected, we noticed that long-standing high blood pressure patients with or without treatment were associated with an increased prevalence of microalbuminuria. We also studied the association of microalbuminuria with the severity and duration of hypertension. We noticed that there was a positive association between microalbuminuria and longstanding hypertensive patients $(\mathrm{P}<0.0001)$. This study had similar findings as the study conducted by Kartik et al. ${ }^{17}$ (Chisquare value of 27.38 and a $\mathrm{P}<0.001$ ) and Stalin et al. ${ }^{13}$ According to Stalin et al. long-standing hypertensives (8.44 \pm 5.58 years) had more increased incidence of microalbuminuria.

There was a huge factual affiliation between the presence of microalbuminuria and patients with grade 2 hypertension 
$(\mathrm{P}=0.001)$. This affiliation was not noted in patients of grade 1 and 3 hypertension. This could be because over $60 \%$ of our study populace had hypertension with grade 2 severity. Comparative discoveries were additionally seen in the review by Stalin et al. ${ }^{13}$

LVH, like microalbuminuria, occurs very early in patients with hypertension ${ }^{18}$ and there is a huge relationship between these two entities - LVH and microalbuminuria as displayed in numerous studies. ${ }^{3,19,20,21}$ The prevalence of LVH found in our study was $52.7 \%$ and is similar to the studies by Kartik et al. ${ }^{16}$ and Stalin et al. ${ }^{13}$ Be that as it may, the prevalence (52.7 $\%$ ) is much more than in a study conducted in 2008 in South India by Hitha et al.14 (29.33\%). The reason for the higher prevalence in our study could be because the study by Hitha et al. involved both outpatients and inpatients whereas our study group included only in-patients who were already admitted because of their poorly controlled blood pressure. A better comparison would have been obtained if the cohort included equal number of inpatients and outpatients.

In opposition to the observations made in the ETODH study, 22 we noticed a strong relationship between hypertensive retinopathy and microalbuminuria. The predominance of microalbuminuria was higher among those with hypertensive retinopathy in this review (51.7\%). The higher predominance of microalbuminuria in patients with hypertensive retinopathy correlated with the study by Stalin et al. ${ }^{13}$ In the review by Busari et al. ${ }^{15}$ advanced hypertensive retinopathic changes, i.e., Grades III-IV were more often seen in patients with microalbuminuria than in those without microalbuminuria (22.6\% versus $1.5 \%)$. This shows that there is a critical relationship between microalbuminuria and target organ damage in essential hypertension and microalbuminuria can be viewed as an early forerunner of target organ damage.

Consequently, this review saw that hypertensive patients with microalbuminuria were more prone to develop target organ damage like $\mathrm{LVH}$ and retinopathy than patients without it. Microalbuminuria would thus be able to serve as a marker of subclinical cardiovascular injury and may account for the worse outcome that is usually associated with increased urinary albumin excretion in essential hypertension. In this way, hypertensive patients with microalbuminuria ought to be assessed for the presence of LVH and retinopathy. These patients are inclined to develop stroke, coronary artery disease, and heart failure. Therefore, aggressive administration of positive cases with drugs that decline microalbuminuria might decrease their higher supposition for development of severe CKD, and adverse cardiovascular events as displayed in numerous studies. $18,19,21,23,24$

\section{CONCLUSIONS}

Most of microalbuminuria positive cases were found appropriated among higher ages. In this study, gender didn't represent a more serious danger for microalbuminuria. There was a huge measurable affiliation present between the microalbuminuria and span and the seriousness of hypertension. There is also a huge measurable affiliation present between microalbuminuria and target organ damage like LVH and retinopathy in hypertensive patients.

Data sharing statement provided by the authors is available with the full text of this article at jemds.com.

Financial or other competing interests: None.

Disclosure forms provided by the authors are available with the full text of this article at jemds.com.

\section{REFERENCES}

[1] Carretero OA, Oparil S. Essential Hypertension. Am Heart J 2000;101(3):329-35.

[2] Oparil S, Acelajado MC, Bakris GL, et al. Hypertension. Nat Rev Dis Primers 2018;4:18014.

[3] Wachtell K, Olsen MH, Dahlöf B, et al. Microalbuminuria in hypertensive patients with electrocardiographic left ventricular hypertrophy: the LIFE study. J Hypertens 2002;20(3):405-12.

[4] Naidoo DP. The link between microalbuminuria, endothelial dysfunction and cardiovascular disease in diabetes. Cardiovasc J S Afr 2002;13(4):194-9.

[5] Wachtell $\mathrm{K}$, Palmieri $\mathrm{V}$, Olsen $\mathrm{MH}$, et al. Urine albumin/creatinine ratio and echocardiographic left ventricular structure and function in hypertensive patients with electrocardiographic left ventricular hypertrophy: the LIFE study. Losartan Intervention for Endpoint Reduction. Am Heart J 2002;143(2):319-26.

[6] Badiger S, Akkasaligar PT, Patil LS, et al. Microalbuminuria in human immunodeficiency virus infection and acquired immunodeficiency syndrome. International Journal of Medical, Health, Biomedical, Bioengineering and Pharmaceutical Engineering 2012;6(8):375-80.

[7] Williams B, Mancia G, Spiering W, et al. 2018 ESC/ESH Guidelines for the management of arterial hypertension. Eur Heart J 2018;39(33):3021-104.

[8] Basi S, Fesler P, Mimran A, et al. Microalbuminuria in type 2 diabetes and hypertension - a marker, treatment target, or innocent bystander? Diabetes Care 2008;31 Suppl 2:S194-201.

[9] Kansal S, Roitman D, Sheffield LT. Interventricular septal thickness and left ventricular hypertrophy. An echocardiographic study. Circulation 1979;60(5):105865.

[10] Bokman CL, Gonzalez MA. Hypertensive retinopathy. Manual of retinal diseases. Springer 2016:627-9.

[11] Poudyal N, Rana KJ, Srivastav B, et al. Frequency of microalbuminuria in hypertensive patients with left ventricular hypertrophy. PMJN Postgraduate Medical Journal of NAMS 2010;10(2):41-4.

[12] Raja BR, Senthamarai A, Anandan H. Microalbuminuria and its correlation with left ventricular hypertrophy and retinopathy in non-diabetic hypertensive patients. Int J Sci Study 2017;5(2):125-9.

[13] Stalin DJ, Benny M, Nandhini S, et al. A clinical study on the effect of telmisartan with alpha lipoic acid on microalbuminuria in type 2 diabetes and hypertensive patients. Int J Pharm Sci Rev Res 2019;57(1):139-42.

[14] Hitha B, Pappachan JM, Pillai HB, et al. Microalbuminuria in patients with essential hypertension and its 
relationship to target organ damage: an Indian experience. Saudi J Kidney Dis Transpl 2008;19(3):4119.

[15] Busari OA, Opadijo OG, Omotoso AB. Microalbuminuria and hypertensive retinopathy among newly diagnosed nondiabetic hypertensive adult Nigerians. Niger J Clin Pract 2011;14(4):436-9.

[16] Hemmati $R$, Gharipour M, Shemirani $H$, et al. Urine albumin to creatinine ratio and echocardiographic left ventricular structure and function in patients with essential hypertension. Am Heart Hosp J 2011;9(2):90-4.

[17] Kartik SV, Manohar S, Siddeswari R, et al. A study of prevalence of micro albuminuria in non-diabetic hypertensives and its correlation with left ventricular mass. J Assoc Physicians India 2014;62:136-42.

[18] Messerli FH, Williams B, Ritz E. Essential hypertension. Lancet 2007;370(9587):591-603.

[19] Cuspidi C, Meani S, Fusi V, et al. Isolated ambulatory hypertension and changes in target organ damage in treated hypertensive patients. J Hum Hypertens 2005;19(6):471-7.
[20] Wachtell K, Ibsen $\mathrm{H}$, Olsen $\mathrm{MH}$, et al. Albuminuria and cardiovascular risk in hypertensive patients with left ventricular hypertrophy: the LIFE study. Ann Intern Med 2003;139(11):901-6.

[21] Assadi F. Effect of microalbuminuria lowering on regression of left ventricular hypertrophy in children and adolescents with essential hypertension. Pediatr Cardiol 2007;28(1):27-33.

[22] Cuspidi C, Meani S, Valerio C, et al. Prevalence and correlates of advanced retinopathy in a large selected hyper-tensive population. The Evaluation of Target Organ Damage in Hypertension (ETODH) study. Blood Press 2005;14(1):25-31.

[23] Arnold JMO, Yusuf S, Young J, et al. Prevention of heart failure in patients in the heart outcomes prevention evaluation (HOPE) study. Circulation 2003;107(9):128490.

[24] Parving HH, Lehnert H, BrochnerMortensen J, et al. The effect of Irbesartan on the development of diabetic nephropathy in patients with type 2 diabetes. $\mathrm{N}$ Engl J Med 2001;345(12):870-8. 